\title{
Precision Medicine for Type 2 Diabetes
}

Anna L Gloyn DPhil ${ }^{1,2,3}$, Daniel Drucker M.D. ${ }^{4,}{ }^{*}$

1. Oxford Centre for Diabetes Endocrinology \& Metabolism, Radcliffe Department of Medicine, University of Oxford, UK

2. Wellcome Centre for Human Genetics, University of Oxford, UK

3. NIHR Oxford Biomedical Research Centre, Churchill Hospital, Oxford, UK

4. The Department of Medicine, Lunenfeld-Tanenbaum Research Institute, Mt. Sinai Hospital, University of Toronto, Toronto, ON Canada M5G 1X5

Address for Correspondence

Dr. Daniel J. Drucker

Mt. Sinai Hospital

600 University Ave TCP5-1004 Mailbox 39

Toronto Ontario

Canada M5G 1X5 drucker@lunenfeld.ca V 416-361-2661 F 416-361-2669 
The study of type 2 diabetes (T2D) has been energized by advances in human genetics, epigenetics, biomarkers, mechanistic studies and large clinical trials, enabling new insights into disease susceptibility, pathophysiology, progression, and development of complications. Simultaneously, multiple new drug classes with different mechanisms of action have been introduced over the last two decades, accompanied by data informing on cardiovascular safety and non-glycemic outcomes. Here we critically examine the progress and integration of this new science into clinical practice, and review opportunities for enabling precision medicine in the diagnosis and treatment of T2D. We contrast the success in delivering personalised medicine for monogenic diabetes with the greater challenging of providing a precision medicine approach for type 2 diabetes highlighting gaps, limitations, and areas requiring further study.

Search strategy and selection criteria

We searched PubMed from January 1 2000-March 22018 for relevant journal articles published in English using query terms including diabetes, genetics, biomarkers, pharmacogenomics, precision medicine, and personalized medicine, from 2010 to 2018. We also searched reference lists of key articles. After reviewing 100 papers, a smaller list of references was selected for inclusion based on quality of data, study sizes, and direct relevance to topic.

Conflicts of interest

DJD is a consultant to Intarcia, Merck, Novo Nordisk Inc, and Pfizer. Mt. Sinai Hospital receives funding for preclinical studies for work in the Drucker lab from GSK, Merck and Novo Nordisk Inc. ALG receives funding for preclinical studies from Novo Nordisk. 
In recent years there has been much interest and it could be argued hype, in our increased ability to use human genetics, lifestyle and environmental information to individualise treatment decisions. Although not a novel concept, the technological advancements which have driven the omics revolution have provided impetus and expectation that a new dawn in "precision" medicine is approaching. In this review we use the term "precision medicine" to describe the stratification of patients into groups based on molecular and genetic biomarkers and clinical characteristics to optimise therapeutic outcomes. The scope of precision medicine has appropriately been broadened to not only include genetics, but to also include environmental and lifestyle factors, with recommendations targeting groups rather than individual patients. In this review we set out to examine the evidence for precision medicine in diabetes focusing on the success in monogenic diabetes, the emerging evidence for type 2 diabetes (T2D) and identifying challenges for enabling more individualized treatment recommendations in the coming years.

\section{Precision medicine in monogenic vs. polygenic diabetes}

The last ten years have seen unprecedented progress in our understanding of the genetic basis of diabetes. With this has come an expectation that our ability to diagnose, characterise and treat individuals with diabetes will be transformed. Given the well documented success in rapidly translating gene discovery efforts for monogenic varieties of diabetes into changes in clinical care pathways, this belief is understandable. Up to $3 \%$ of diabetes diagnosed in children has a monogenic basis with the most common cause mutations in the transcription factor gene hepatocyte nuclear factor 1 alpha $(H N F 1 A)^{1}$. Individuals with rare penetrant mutations in HNF1A typically present before the age of 25 years with non-insulin dependent diabetes, are slim and have a strong family history of diabetes. Clinical investigation usually reveals that they are autoantibody negative but C-peptide positive. An early case study highlighted that individuals with diabetes due to an HNF1A mutation had marked sensitivity to sulphonylureas (SUs), a finding that was elegantly substantiated in a randomised controlled trial providing the first example of personalised medicine in diabetes ${ }^{2}$. The precise molecular mechanism for sensitivity to this class of oral hypoglycaemic agent remains elusive but it has been argued that closure of the $\mathrm{K}_{\text {ATP }}$ channel bypasses the major sites of $\beta$-cell dysfunction thus reigniting insulin secretion. SUs are also the first line treatment for individuals with neonatal diabetes due to activating mutations in the $K C N J 11$ and $A B C C 8$ genes which cause failure of the $\mathrm{K}_{\text {ATP }}$ channels to close in response to ATP generated by glycolysis ${ }^{3-6}$. Closure of the channel by an ATP-independent mechanism circumnavigates the cause of the $\beta$-cell dysfunction, restoring insulin secretion in these individuals and remarkably improving their glycaemic control. Personalised medicine in monogenic diabetes extends beyond treatment response: for example individuals with loss-of -function mutations in the glucokinase $(G C K)$ gene have stable fasting hyperglycaemia and are unlikely to develop diabetic complications ${ }^{7}$. For neonatal diabetes, non-selective genetic testing now informs on the future development of additional clinical features, disease management and likelihood of disease remission ${ }^{8}$.

At this point however, genetic discovery efforts for T2D have had limited translational benefits. Reconciling the very different "bench-to-bedside" trajectories for monogenic and more complex forms of diabetes can be achieved by appreciating the differences in the genetic contributions to the development of diabetes, which makes the eventual classification of T2D into multiple discrete subtypes 
less likely (Figure 1). As the field has tried to wrestle with how genetics might be used to support precision medicine a number of theories have been proposed for complex diseases such as T2D or coronary artery disease. Two untested models which have garnered interest propose that individuals are very likely to have a diverse set of overlapping mechanisms for their failure to regulate their blood glucose or lipid levels and that these "blends" will be hard to tease apart into discrete subtypes. Both models propose that what matters is where an individual sits on a series of hypothetical "sliders" for particular pathophysiological processes (e.g. beta-cell mass, insulin sensitivity, incretin response) ${ }^{9,10}$. The argument is that if we can understand the composition of the pallet or blend and where individuals sit on these sliding scales of intermediate phenotypes there is the potential to make more tailored treatment choices particularly with identifying patients who are unlikely to respond to a particular therapy. For example people with diabetes largely due to loss of $\beta$-cell mass are unlikely to respond to agents which potentiate insulin secretion. There is already evidence for this hypothesis from studies of the reduced efficacy of GLP-1 receptor agonists (GLP-1RAs) tested in insulin-treated patients with low Cpeptide levels and auto-antibodies ${ }^{11}$. Whilst encouraging evidence for the clinical utility of genetic risk profiling has also emerged from efforts to understand the frequency of T1D in individuals presenting with diabetes in adulthood. Distinguishing these people from those with T2D is important, as individuals with T1D will rapidly require insulin therapy ${ }^{12}$.

\section{Stratification of diabetes into multiple subtypes}

A recent study from Scandinavia has provided support that identifying discrete subtypes of diabetes which have different compositions of pathophysiology may inform of disease trajectory. By performing hierarchical clustering in around nine thousand newly diagnosed individuals with diabetes, based on clinical variables (auto-antibodies, age at diagnosis, BMI, $\mathrm{HbA1c}$, and estimates of $\beta$-cell function and insulin resistance) they were able to identify 5 sub-types of diabetes which replicated in independent cohorts ${ }^{13}$. In support of different pathophysiological features the clustering of established genetic associations differed between these classes. In line with different degrees of shared pathophysiology the risks of complications were different across the classes. Notably, diabetic retinopathy was identified earlier in subjects with relative insulin deficiency, whereas insulin-resistant individuals exhibited a greater risk of developing diabetic kidney disease ${ }^{13}$. These findings, if replicated in ethnically diverse populations across a range of ages, may be of potential value for clinical trial enrollment or for early treatment stratification, perhaps with agents such as SGLT2 inhibitors, that exhibit potential for modification of renal outcomes ${ }^{14}$. Future studies will now need to establish whether treatment response with different drug classes differs across these subclasses and whether they can further be improved and refined by additional prospective omic and clinical data

\section{Pharmacogenomics and clinical trial data inform personalized treatment recommendations}

Notwithstanding tremendous advances in application of genetics to the characterization of diabetes pathogenesis and treatment, the precise etiology of T2D is not currently informed by genetic testing or available biomarkers. Ideally, evidence would include a cost-effective genetic test, coupled with robust prospective validation of the predictive value of incorporating genetic information into the treatment algorithm. Presently, the extent to which genetic variation in the G6PD locus underestimates the 
baseline $\mathrm{HbA} 1 \mathrm{c}$ in some African Americans ${ }^{15}$, mandates consideration of routine genotyping at this locus for black subjects where interpretation of $\mathrm{HbA1c}$ values assumes clinical relevance. Despite tremendous progress in the integrated analysis of tissue and systemic metabolic networks perturbed in the setting of obesity, insulin, resistance, and $\mathrm{T}^{2} \mathrm{D}^{16}$, a uniform set of genetic information or biomarkers providing clinically useful information enabling individualized therapeutic choices has not yet been validated in randomized prospective clinical trials. Hence, a personalized approach to therapy requires consideration of the available clinical trial evidence, complemented by decisions surrounding affordability, patient goals and preferences.

It is important to be as critical of the emerging data for pharmacogenetics studies as we are now of association studies for T2D-risk and to recognise the importance of replication. The vast majority of the current literature is focused on small candidate gene and/or single nucleotide polymorphism (SNP) studies as opposed to unbiased genome-wide assessments of the impact of genetic variation on therapy response. Thus, whilst many studies report associations of T2D-risk alleles and response to treatment, the vast majority of these associations have not yet been replicated in larger prospective studies.

Metformin is the most widely used and intensively studied drug recommended for first line therapy in the treatment of T2D. Indeed, the study of metformin includes a large pharmacogenomics effort analyzing genetic determinants of metformin transport, and molecular targets transducing metformin action. Genetic determinants of variability in metformin pharmacokinetics, tissue uptake and clearance have been identified (SLC22A1, SLC22A2 and SLC47A), however their clinical utility in predicting metformin responsivity in human subjects with T2D remains suboptimal ${ }^{17}$. Some studies have also identified genetic variation within SLC22A1, together with use of medications that inhibit the OCT1 protein, as factors predicting up to a 4-times higher rate of gastrointestinal metformin intolerance ${ }^{17}$. Genetic variation (rs11212617) near the ATM locus is associated with the glycemic response to metformin in some but not all populations however the effect size is modest, precluding current utility in the clinic. More encouraging data, including analysis of gene expression in human liver samples, implicated variation within an intron (rs8192675) of SLC2A2 encoding GLUT2, together with hepatic SLC2A2 expression informing the therapeutic response to metformin in study subjects of European ancestry (up to $0.33 \%$ ( $2.1 \mathrm{mmol} / \mathrm{L}$ ) difference in $\mathrm{HbA} 1 \mathrm{c}$ reduction $)^{17}$. Nevertheless, no association of variation within ATM or SLC2A2 and therapeutic metformin responsivity was detected in human subjects with prediabetes followed in the Diabetes Prevention Program (DPP) trial, highlighting challenges in extrapolating genetic findings from analysis of established T2D to different study populations and metabolic states ${ }^{18}$. Moreover, treatment with metformin or lifestyle improved insulin sensitivity in the DPP study independent of the genetic burden for insulin resistance, based on the presence or absence of 17 established genetic variants associated with insulin sensitivity ${ }^{19}$. Hence although considerable progress has been made in understanding the genetic basis underlying therapeutic responsivity to metformin, the predictive insights to date have been modest. Considering that metformin is inexpensive and associated with a very low risk of major side effects, broad implementation of pharmacogenomic analyses for clinical prediction of metformin efficacy and tolerability is not yet practical, nor cost effective. Continued efforts to understand genetic factors 
underlying differences in therapeutic response between individuals may however shed light on the mechanisms by which metformin improves glycemic control, which continue to be debated ${ }^{20}$.

The historical and ongoing extensive use of sulphonylureas (SUs) and insulin as second line glucoselowering agents, recommended in many T2D guidelines, reflects decades of study and clinical experience. Moreover, intensive glucose control for several years in regimens including these agents is generally associated with reduction of microvascular outcomes over shorter time periods, with longer post-trial follow-up periods, out to 10 years, demonstrating reduction in cardiovascular events and in some trials, decreased all-cause mortality ${ }^{21,22}$. Nevertheless, the relative reduction in rates of microvascular disease may vary considerably depending on the population studied, the precise treatment regimens and duration of observation ${ }^{23,24}$. The use and benefits associated with SUs and insulin is offset by higher rates of hypoglycemia and weight gain ${ }^{24-26}$. Indeed the risk of severe hypoglycemia with these agents may be characterized by loss of consciousness, seizures, changes in mental status, and associated neurological and cardiovascular sequelae, resulting in increased rates of emergency room visits, hospitalization, morbidity and mortality, notably in older patients ${ }^{27,28}$.

Probably the best studied example of genetics informing pharmacological responses is variation at the TCF7L2 locus, where data from the GoDARTS study has shown that carriers of the TCF7L2 T2D risk allele are less likely to achieve glycaemic goals in response to SUs than non-carriers, a treatment effect that was not observed for metformin ${ }^{29}$. Other pharmacogenetic determinants contributing to SU responsivity and failure have also been studied, encompassing genetic variation within genes important for SU metabolism and the SU receptor, $\beta$-cell function, and insulin action, including CYP2C9, ABCC8, KCNJ11, IRS1, CDKAL1, CDKN2A/2B, KCNQ1 and NOS1AP ${ }^{17,30}$. Of particular interest is the case of genetic variation at the $S U$ receptor itself, where a number of studies have investigated whether coding variants in the KCNJ11 and $A B C C 8$ genes which are themselves associated with T2D-risk also influence response to $\mathrm{SU}$ treatment ${ }^{31-33}$. Supported by functional studies there is data to support pharmacogenetic effects on treatment for carriers of the ABCC8 A1369AS variant with a particular subclass of SU (gliclazide, an Asite SU) ${ }^{34}$. Nevertheless, the importance of determining these genotypes for guiding individual SU selection or dose to optimize achievement of clinical T2D outcomes, has not been sufficiently validated in prospective randomized studies.

Notwithstanding concerns surrounding relative benefit vs. risk of thiazolidinediones for glucose control in subjects with T2D, these drugs attenuate disease progression in subsets of subjects with non-alcoholic fatty liver and non-alcoholic steatosis in studies up to 24 months duration ${ }^{35}$. Furthermore pioglitazone reduced the development of new T2D and decreased the rate of fatal or nonfatal stroke or myocardial infarction over 4.8 years in human subjects with insulin resistance and established cerebrovascular disease ${ }^{36}$. Nevertheless, our understanding of the genetic determinants underlying beneficial or adverse therapeutic responses to thiazolidinediones, principally rosiglitazone and pioglitazone, remains limited. Variants within SLCO1B1, encoding the OATP1B1 (Organic Anion Transporting Polypeptide 1B1) transporter influenced glycemic response to rosiglitazone in 833 Scottish subjects assessed over 1-18 months of therapy, whereas variation within CYP2C8, encoding cytochrome P450 2C8 metabolizing enzyme, associated with glycemic response and weight gain ${ }^{37}$. Surprisingly, the presence of these genetic variants did not similarly impact the response to pioglitazone therapy. Moreover, findings that 
genetic variation with PPARG, specifically Pro12Ala, associate with decreased responsivity to thiazolidinediones in some small Asian cohorts, reviewed $\mathrm{in}^{17}$, have not been independently verified in larger heterogeneous populations followed for longer periods of time, hence the broad clinical relevance of these associations remains uncertain.

Acarbose, an $\alpha$-glucosidase inhibitor widely used in some parts of Asia (particularly China), and to a lesser extent elsewhere, reduced development of new incident T2D in humans with impaired glucose tolerance (IGT), and a secondary analysis associated acarbose therapy in this population with reduction of cardiovascular events ${ }^{38}$. Analysis of the STOP-NIDDM trial revealed associations between genetic variation in PPARA, HNF4A, LIPC, PPARG2, and PPARGC1A and the response to acarbose in subsets of subjects with $\mathrm{IGT}^{39}$. Nevertheless, these associations were quantitatively modest, have not yet been confirmed in independent populations, and have not been examined in acarbose-treated human subjects with established T2D. Moreover, an independent study (6,522 subjects, median follow-up of 5 years) assessing the effect of acarbose vs. placebo in human subjects with IGT and established coronary artery disease did not reveal differences in rates of cardiovascular disease, all-cause mortality, hospital admission for heart failure, or impaired renal function ${ }^{40}$.

The utilization of patient-appropriate $\mathrm{HbA} 1 \mathrm{c}$ targets remains a cornerstone of T2D management, however personalized treatment goals beyond $\mathrm{HbA} 1 \mathrm{c}$ based on available clinical evidence and expert opinion are increasingly within reach (Figure 2). The failure to achieve uniform benefit in the Action to Control Cardiovascular Risk in Diabetes (ACCORD) trial provides a note of caution in regard to formulation of $\mathrm{HbA} 1 \mathrm{c}$ targets and implementation of intensive treatment regimens for older subjects with T2D, notably those at risk for cardiovascular disease ${ }^{41}$. Analysis of common genetic variants from white individuals with T2D in the ACCORD trial identified two genetic variants significantly associated with cardiovascular mortality in response to intensive diabetes therapy ${ }^{42}$.

A genetic risk score based on these variants was further validated using the clinical care T2D population at the Joslin clinic, and subjects studied in the Outcome Reduction with an Initial Glargine Intervention (ORIGIN) trial ${ }^{26}$. Remarkably, a 22\% increase in plasma GLP-1 levels in a subset of intensively-treated subjects in ACCORD harboring the C/C variant of rs57933 was associated with a favorable GRS and cardiovascular benefit ${ }^{43}$. The implications of these findings and applicability of this risk score to individualization of T2D therapy for younger subjects utilizing treatment regimens that minimize hypoglycemia remains uncertain and requires prospective validation.

Three new classes of glucose-lowering agents, GLP-1RAs, dipeptidyl peptidase-4 (DPP4) inhibitors and sodium glucose cotransporter-2 inhibitors (SGLT2i) have been introduced which extend our ability to treat T2D with reduced rates of hypoglycemia, less frequent self-blood glucose monitoring, simplified dosing regimens, and without weight gain. These drugs were initially developed based on understanding of metabolic physiology ${ }^{44-46}$, with mechanism of action and safety of SGLT2i and GLP-1RA subsequently informed through analysis of genetic variation at these loci in individuals ${ }^{47}$ and large population studies ${ }^{48}$. Reinforcing the ability of human genetics to identify safe and effective therapeutic targets, arraybased studies focused on assessing coding variation exome-wide identified a low-frequency nonsynonymous variant A316T in the receptor for GLP-1 which associates with fasting glucose levels ${ }^{49}$, 
50. The glucose-lowering allele is also associated with decreased T2D risk yet a paradoxical reduction in the early $\beta$-cell response to glucose, quantified by insulinogenic index ${ }^{50}$. Furthermore, and pertinent for therapeutic response, the glucose- lowering allele is associated with protection against coronary heart disease ${ }^{48}$. Nevertheless, the extent to which genetic variation within the GLP1R contributes to glycemic or non-glycemic outcomes in clinical trials employing GLP-1R agonists is not yet known. Even less is known about the pharmacogenetic determinants underlying the glycemic response to DPP4 or SGLT2 inhibitors. Genetic variation at rs7202877 near CTRB1/2, a known diabetes risk locus, associated with differential insulin secretory responses to acute GLP-1 infusion and also associated with a lower A1C response to DPP-4 inhibitor treatment ${ }^{51}$. Genetic variation and clinical biomarkers associating with declining $\beta$-cell function also predicts the response to GLP-1R agonist therapy in some populations ${ }^{11}$. Recent findings from the Predicting Response to Incretin Based Agents (PRIBA) study, validated independently using case records from the Clinical Practice Research Datalink, support the use of baseline clinical characteristics in decisions on incretin based therapies. Individuals with markers of insulin resistance such as higher fasting C-peptide, HOMA2 insulin resistance and triglycerides responded less well over a 6 month treatment period to DPP-4 inhibitor treatment but their response to GLP-1 RA was unaffected ${ }^{52}$.

Considerable effort has been expended, using genetics, biomarkers, metabolomics, and gut microbial profiling to prospectively identify ideal candidates for metabolic surgery with a greater likelihood of achieving sustained diabetes remission. Development of a Diabetes Remission (DiaRem) scoring system (age, HbA1c, use of specific glucose-lowering medications) was followed by introduction of an Advanced DiaRem (Ad-DiaRem) score, refined through addition of diabetes duration and number of glucoselowering agents. Ad-DiaRem enhanced predictive accuracy for diabetes remission at 1 year in severely obese individuals ${ }^{53}$. These clinical tools, while promising, require further validation in longer prospective clinical trials, as the rate of T2D remission after surgery drops progressively with follow-up beyond 1 year. Moreover, extending these analyses from Roux-en-Y (RYGB) Gastric Bypass to vertical sleeve gastrectomy, potentially incorporating indices reflecting $\beta$-cell function, tissue fibrosis, and additional metabolic profiling data, may enhance the performance and utility of emerging predictive scoring systems. The available observational data support a sustained improvement in blood pressure and control of dyslipidemia and a 51\% rate of T2D remission assessed 12 years after RYGB ${ }^{54}$. RYGB surgery reduced the rates of cardiovascular death in obese subjects followed over time in the Swedish Obese Subjects study, however study subjects were at least 37 years old at the time of surgery, and only $7.4 \%$ of individuals had $\mathrm{T}^{2} \mathrm{D}^{55}$. Nevertheless, the incidence of myocardial infarction, but not stroke, was reduced in obese subjects with $T 2 D^{56}$.

\section{Evidence guiding therapeutic recommendations for T2D}

Optimization of diet and exercise remain key initial therapeutic recommendations for virtually all patients with T2D, together with treatment of co-existing blood pressure, dyslipidemia, and consideration of anti-platelet therapy. Despite the explosion of genetic and biomarker information refining our understanding of the underlying characteristics of T2D in different populations, regulatory guidance for T2D currently embodies only a single precision medicine recommendation. Health care providers should consider genetic testing for glucose-6-phosphate dehydrogenase (G6PD) deficiency 
prior to the use of some SUs ${ }^{57}$, given the risk of hemolytic anemia. Hence, a personalized medicine approach to T2D mandates extensive consideration of clinical trial data, in the context of individual patient goals and characteristics.

In the absence of robust comparative effectiveness data, and limited insights from prospective use of genetic and biomarker studies to inform long term therapeutic efficacy, evidence-based formulation of personalized treatment recommendations for patients with T2D is challenging. Randomized controlled trials comparing individualized treatment regimens vs. traditional sequential therapy based on $\mathrm{HbA} 1 \mathrm{c}$ targets are limited. Clearly, each patient is unique, with different goals, medical history, and expectations (Figure 2). Moreover, medication access and cost vary widely, and substantially influence individual treatment recommendations. Although some expert guidance advocates initial therapy with two or more glucose lowering medications for individuals with higher levels of $\mathrm{HbA1c}$ to counteract clinical inertia, long term studies and evidence supporting early initial combination therapy vs. sequential addition of medications is lacking ${ }^{58}$.

\section{Application of Cardiovascular Outcome Trials (CVOTs) to Clinical Practice}

Two classes of drugs, the SGLT2 inhibitors (SGLT2i), and GLP1R agonists (GLP-1RAs), notably liraglutide, have been associated with reduction of major adverse cardiac events (MACE) and reduced mortality in subjects with T2D studied in randomized cardiovascular outcome trials (CVOTs). The EMPA-REG OUTCOME trial enrolled subjects $>18$ years of age with established cardiovascular disease (CVD) ${ }^{59}$. The population studied in the Liraglutide Effect and Action in Diabetes: Evaluation of Cardiovascular Outcome Results (LEADER) trial was at least 50 years old at the time of study enrollment, with 80\% of enrolled subjects exhibiting prior $C V D^{60}$. Subgroup analyses of EMPA-REG OUTCOME revealed that as a group, African-American subjects, or individuals $<65$ years old, or those with a $\mathrm{BMI}>30 \mathrm{~kg} / \mathrm{m}^{2}$ did not exhibit reductions in the primary cardiovascular outcome, but all three subgroups exhibited a reduced rate of cardiovascular death ${ }^{59}$. Less than $4 \%$ of subjects enrolled in the Canagliflozin Cardiovascular Assessment Study (CANVAS) program were black or African American, and individuals less than age 50 were not included in the primary prevention cohort, limiting direct application of evidence from CANVAS to patient $\mathrm{C}^{61}$. Nevertheless, the reduction in cardiovascular events detected with canagliflozin provides reassuring evidence for the cardiovascular safety associated with SGLT2i therapy.

Although results of CVOTs have provided substantial insight into the benefits and safety of newer antidiabetic agents, extrapolation of results from CVOTs to broader T2D populations is problematic. It is currently unknown whether initiation of therapy with SGLT2 inhibitors or GLP-1R agonists in healthier subjects with T2D earlier in the course of the disease will provide substantial cardiovascular benefit with chronic therapy. Indeed subgroup analysis in LEADER revealed lack of benefit in the cohort of subjects > 60 years of age without established CVD ${ }^{60}$. Many CVOTs enrolled relatively few female subjects over the age of 75 and small numbers of subjects over the age of 80 . Ideally, treatment recommendations and targets for elderly subjects need to be adjusted based on life expectancy and individualized based on the relative risk of developing new diabetes complications over defined time periods ${ }^{62}$. The development of polygenic risk scores enabling identification of individuals at high risk for development of coronary artery disease $^{63}$ may prove useful for selecting individuals without known CVD for earlier treatment with 
SGLT2i or GLP-1R agonists; however information on the predictive value of these scores in large populations of subjects with T2D, independent of aggressive treatment of blood pressure and dyslipidemia, is currently limited.

\section{Targeting glycemic outcomes and complications beyond HbA1c}

Current evaluation of therapeutic effectiveness remains predominantly focused on a single glycemic measure, $\mathrm{HbA} 1 \mathrm{c}$, however it seems likely that this will change. The expanding use of CGM to refine understanding of the importance of glycemic parameters beyond $\mathrm{HbA} 1 \mathrm{c}$, including rates of hypoglycemia, and proportions of time within target glucose ranges, is receiving increasing attention in the management of both T1D and T2D. Notably, HbA1c may not always reflect or predict the burden of disease, and CGM may be particularly useful for individuals with unexplained recurrent hypoglycemia or marked glycemic variability. A limited assessment using several capillary blood glucose determinations each month correlated the extent of glycemic variability with rates of severe hypoglycemia in the Trial Comparing Cardiovascular Safety of Insulin Degludec versus Insulin Glargine in Patients with Type 2 Diabetes at High Risk of Cardiovascular Events (DEVOTE) ${ }^{64}$. Moreover, the development of severe hypoglycemia correlated temporally with rates of all-cause mortality in subjects studied in DEVOTE ${ }^{65}$. More information on the potential clinical importance of severe hypoglycemia is likely forthcoming from imminent results of the CARdiovascular Outcome Trial of LINAgliptin Versus Glimepiride in Type 2 Diabetes (CAROLINA) trial ${ }^{66}$, which may, depending on the results, further shift the T2D treatment paradigm away from medicines associated with an increased risk of severe hypoglycemia, most notably in elderly individuals and subjects at high risk for cardiovascular events (Figure 2). Hence future precision diabetes initiatives will likely encompass newer goals and non-glycemic targets, once these become prospectively validated.

Individualization of therapeutic options beyond metformin, as outlined in Figure 2, largely reflects expert opinion, not prospective validation in randomized trials. The demonstration that several SGLT2i and at least one GLP-1RA reduced MACE events further refines current options for reduction of heart failure (SGLT2i) and MACE-related complications (GLP-1RA and SGLT2i) in subjects with T2D. Although heart failure has not been a traditional focus for adjudication of CVD events in CVOTs undertaken in subjects with T2D, a reduction in hospitalization for heart failure was reported in both the EMPA-REG OUTCOME and CANVAS trials, highlighting the importance of heart failure as an independent outcome in subjects with $T 2 \mathrm{D}^{59,61}$. Moreover, a reduction in progression of diabetic nephropathy, including doubling of serum creatinine and time to renal replacement, was detected in subjects treated with empagliflozin in the EMPA-REG OUTCOME trial ${ }^{14}$. Similarly, a numerical reduction of renal events, although not statistically significant, was demonstrated in canagliflozin-treated subjects in the CANVAS trial ${ }^{61}$.

Less compelling, but equally intriguing, are exploratory studies demonstrating reduction of liver fat and attenuation of liver inflammation and fibrosis in subjects with prediabetes or T2D treated with $45 \mathrm{mg}$ daily of pioglitazone for 18-36 months ${ }^{67}$. Moreover a smaller, double-blind, randomized controlled trial of liraglutide $1.8 \mathrm{mg}$ daily for 48 weeks, including $35 \%$ of subjects with T2D, revealed a reduction in the extent of NASH and fibrosis in liraglutide-treated subjects ${ }^{68}$. While much larger registration studies will 
be needed to validate the preliminary findings for these putative non-glycemic indications, it seems likely that therapeutic recommendations for the management of T2D will increasingly be based on a personalized combination of goals encompassing both glycemic and non-glycemic targets (Figure 2), based on accumulating evidence from outcome and registration studies.

\section{Mind the Gap in Personalized Medicine}

Despite the introduction of multiple new drug classes for the treatment of T2D, data from real world health care audits demonstrates that population $\mathrm{HbA1c}$ levels have failed to decline as much as might be expected based on analysis of clinical trial data. Although many explanations, encompassing barriers to medication and health care access, contribute to this discrepancy, poor medication adherence underlies the challenge in achieving target $\mathrm{HbA} 1 \mathrm{cs}$ for many patients ${ }^{69}$. Despite substantial differences in HbA1c outcomes favoring GLP-1RAs over DPP-4 inhibitors in multiple clinical trials, retrospective claims analysis revealed comparable (and disappointing) $\mathrm{HbA} 1 \mathrm{c}$ reductions of $\sim 0.51 \%$ for both classes, with very low rates of medication adherence (29-37\% for GLP-1RAs vs. DPP-4 inhibitors, respectively) underlying the results achieved ${ }^{70}$. The introduction of medications taken once weekly or even less frequently may theoretically improve compliance with diabetes therapies, however this concept requires rigorous prospective validation in real world studies.

Poor adherence is not limited to glucose-lowering agents, but extends to therapies directed at reduction of blood pressure, platelet aggregation, and dyslipidemia. As poor adherence is associated with increased rates of hospitalization and all-cause mortality in subjects with $T 2 \mathrm{D}^{71}$, understanding the myriad causes underlying adherence in different individuals is critical for successful application of personalized diabetes therapy. Intensification of efforts directed at identifying key factors underlying the success of RCTs (frequent interaction with health care providers, finite, time-limited and incentivized commitments to clinic visits and compliance, unencumbered access to medications, extensive provision of resources and regular support) and implementing best practices in the clinic represents a major challenge and opportunity for improved delivery of effective diabetes care in the real world.

Multiple international studies investigating patient stratification or predictors of response to therapy in treatment naïve individuals are underway including the Innovative Medicines Initiative funded DIRECT study (http://www.direct-diabetes.org/), the MASTERMIND and SUGAR-MGH studies ${ }^{72,73}$. These efforts

will help address some of the current gaps in our knowledge regarding the impact of metabolic state on response to treatment. Indeed recent results from the SUGAR-MGH study demonstrate a role for the TCF7L2-T2D risk alleles in the acute responses to both glipizide and metformin in people without diabetes $^{74}$. Whether determination of these risk alleles provides similarly useful information on the chronic response to therapy in subjects with T2D remains uncertain.

\section{Future Directions, Limitations, Recommendations, Areas of Uncertainty}

The available evidence strongly links early and sustained control of T2D with long term benefit and reduction of complications. Greater use of electronic medical record-generated information for monitoring achievement of blood pressure, glucose, weight, and lipid targets, together with timely reminders for frequency of eye exams and foot care, should reduce the morbidity and mortality 
associated with T2D. It is early days in our understanding of how genetic information can be used to stratify individuals for treatment response and it is almost certain that this information will need to be considered in concert with the clinical status of each individual along their disease progression path. The increasing availability and decreasing cost of human genetic analysis makes it likely that precise genome analyses will become routine in clinical medicine, and utilized for diagnosis and therapeutic recommendations in multiple subspecialties beyond the diabetes clinic. Refinement of algorithms incorporating predictive genetic variation and biomarkers for drug responsivity and the risk of complications, prospectively validated by clinical trial outcomes data in multiple populations with different ethnic backgrounds, should progressively enhance our ability to transform diabetes care. Although there is much more progress to come, it is not farfetched to anticipate gradual implementation of cost-effective precision-based personalized medicine in T2D treatment centers.

Successful deployment of a precision medicine approach to T2D requires attention to gaps in our current knowledge base, including a paucity of clinical trial data guiding treatment in adolescents, the elderly, and during pregnancy. Moreover, the majority of study subjects in trials informing the safety and cardiovascular benefit of new medications for T2D were recruited with established cardiovascular disease. Our outcomes data for recommending T2D therapy directed at primary prevention of macrovascular complications in younger healthier individuals is insufficient. It seems certain that the increasing availability, and improved accuracy and utility of genomic and clinical biomarkers, will further enable precision diabetes. Simultaneously, information technology will continuously improve our capacity to conduct global, large scale and cost-effective clinical trials. Nevertheless, given the staggering numbers of subjects with T2D, critical evaluation of the cost vs. benefit for utilization of genomics, biomarkers, new technologies, and specific medications will be needed to support recommendations for clinical use in specific populations. Given the tremendous progress made over the last decade, it is reasonable to predict greater adoption of precision medicine approaches in the T2D clinic in the years to come.

\section{Acknowledgements}

ALG is a Wellcome Trust Senior Fellow in Basic Biomedical Research (095101/Z/10/Z and 200837/Z/16/Z). DJD is supported by the Canada Research Chairs program a Banting and Best Diabetes Centre Novo Nordisk Chair in Incretin Biology, and CIHR grant 154321

\section{Figures}

Figure 1: Illustration of current status of precision medicine approaches for treatment of individuals with selected monogenic subtypes of diabetes (left panel) vs. individuals with complex genetic determinants of T2D (right panel) 
Figure 2: Evolving clinical considerations underlying personalized medicine recommendations for intensification of therapy after metformin in subjects with T2D. Clinical characteristics influencing medication selection (left panel) vs. recent evidence from clinical studies examining the non-glycemic actions of glucose-lowering therapies on complications associated with T2D (right panel). OAD Orally administered anti-diabetic drugs; NAFLD, non-alcoholic fatty liver disease; NASH, non-alcoholic steatohepatitis; $\mathrm{HF}=$ heart failure; CAD, coronary artery disease; CVA, cerebrovascular accident; CKD, chronic kidney disease. 
1. Shepherd M, Shields B, Hammersley S, Hudson M, McDonald TJ, Colclough K, et al. Systematic Population Screening, Using Biomarkers and Genetic Testing, Identifies 2.5\% of the U.K. Pediatric Diabetes Population With Monogenic Diabetes. Diabetes care. 2016; 39(11): 1879-88.

2. Pearson ER, Starkey BJ, Powell RJ, Gribble FM, Clark PM, Hattersley AT. Genetic cause of hyperglycaemia and response to treatment in diabetes. Lancet. 2003; 362(9392): 1275-81.

3. Gloyn AL, Pearson ER, Antcliff JF, Proks P, Bruining GJ, Slingerland AS, et al. Activating mutations in the gene encoding the ATP-sensitive potassium-channel subunit Kir6.2 and permanent neonatal diabetes. The New England journal of medicine. 2004; 350(18): 1838-49.

4. Pearson ER, Flechtner I, Njolstad PR, Malecki MT, Flanagan SE, Larkin B, et al. Switching from insulin to oral sulfonylureas in patients with diabetes due to Kir6.2 mutations. The New England journal of medicine. 2006; 355(5): 467-77.

5. Babenko AP, Polak M, Cave H, Busiah K, Czernichow P, Scharfmann R, et al. Activating mutations in the $A B C C 8$ gene in neonatal diabetes mellitus. The New England journal of medicine. 2006; 355(5): 456-66.

6. Rafiq M, Flanagan SE, Patch AM, Shields BM, Ellard S, Hattersley AT. Effective treatment with oral sulfonylureas in patients with diabetes due to sulfonylurea receptor 1 (SUR1) mutations. Diabetes care. 2008; 31(2): 204-9.

7. Steele AM, Shields BM, Wensley KJ, Colclough K, Ellard S, Hattersley AT. Prevalence of vascular complications among patients with glucokinase mutations and prolonged, mild hyperglycemia. Jama. 2014; 311(3): 279-86.

8. De Franco E, Flanagan SE, Houghton JA, Lango Allen H, Mackay DJ, Temple IK, et al. The effect of early, comprehensive genomic testing on clinical care in neonatal diabetes: an international cohort study. Lancet. 2015; 386(9997): 957-63.

9. McCarthy MI. Painting a new picture of personalised medicine for diabetes. Diabetologia. 2017; 60(5): 793-9.

10. Khera AV, Kathiresan S. Is Coronary Atherosclerosis One Disease or Many? Setting Realistic Expectations for Precision Medicine. Circulation. 2017; 135(11): 1005-7.

11. Jones AG, McDonald TJ, Shields BM, Hill AV, Hyde CJ, Knight BA, et al. Markers of beta-Cell Failure Predict Poor Glycemic Response to GLP-1 Receptor Agonist Therapy in Type 2 Diabetes. Diabetes care. 2016; 39(2): 250-7.

12. Thomas NJ, Jones SE, Weedon MN, Shields BM, Oram RA, Hattersley AT. Frequency and phenotype of type 1 diabetes in the first six decades of life: a cross-sectional, genetically stratified survival analysis from UK Biobank. The Lancet Diabetes \& Endocrinology. 2017.

13. Ahlqvist E, Storm P, Käräjämäki A, Martinell M, Dorkhan M, Carlsson A, et al. Novel subgroups of adult-onset diabetes and their association with outcomes: a data-driven cluster analysis of six variables. The Lancet Diabetes \& Endocrinology. 2018; 10.1016/s2213-8587(18)30051-2.

14. Wanner C, Inzucchi SE, Lachin JM, Fitchett D, von Eynatten M, Mattheus M, et al. Empagliflozin and Progression of Kidney Disease in Type 2 Diabetes. The New England journal of medicine. 2016; 375(4): 323-34.

15. Wheeler E, Leong A, Liu CT, Hivert MF, Strawbridge RJ, Podmore C, et al. Impact of common genetic determinants of Hemoglobin A1c on type 2 diabetes risk and diagnosis in ancestrally diverse populations: A transethnic genome-wide meta-analysis. PLoS medicine. 2017; 14(9): e1002383.

16. Nielsen J. Systems Biology of Metabolism: A Driver for Developing Personalized and Precision Medicine. Cell metabolism. 2017; 25(3): 572-9.

17. Zhou K, Pedersen HK, Dawed AY, Pearson ER. Pharmacogenomics in diabetes mellitus: insights into drug action and drug discovery. Nature reviews Endocrinology. 2016; 12(6): 337-46. 
18. Florez JC. Pharmacogenetics in type 2 diabetes: precision medicine or discovery tool? Diabetologia. 2017; 60(5): 800-7.

19. Hivert MF, Christophi CA, Franks PW, Jablonski KA, Ehrmann DA, Kahn SE, et al. Lifestyle and Metformin Ameliorate Insulin Sensitivity Independently of the Genetic Burden of Established Insulin Resistance Variants in Diabetes Prevention Program Participants. Diabetes. 2016; 65(2): 520-6.

20. Rena G, Hardie DG, Pearson ER. The mechanisms of action of metformin. Diabetologia. 2017; 60(9): 1577-85.

21. Holman RR, Paul SK, Bethel MA, Matthews DR, Neil HA. 10-year follow-up of intensive glucose control in type 2 diabetes. The New England journal of medicine. 2008; 359(15): 1577-89.

22. Hayward RA, Reaven PD, Wiitala WL, Bahn GD, Reda DJ, Ge L, et al. Follow-up of glycemic control and cardiovascular outcomes in type 2 diabetes. The New England journal of medicine. 2015; 372(23): 2197-206.

23. Duckworth W, Abraira C, Moritz T, Reda D, Emanuele N, Reaven PD, et al. Glucose control and vascular complications in veterans with type 2 diabetes. The New England journal of medicine. 2009; 360(2): 129-39.

24. Patel A, MacMahon S, Chalmers J, Neal B, Billot L, Woodward M, et al. Intensive blood glucose control and vascular outcomes in patients with type 2 diabetes. The New England journal of medicine. 2008; 358(24): 2560-72.

25. Group UPDSU. Intensive blood-glucose control with sulphonylureas or insulin compared with conventional treatment and risk of complications in patients with type 2 diabetes (UKPDS 33). UK Prospective Diabetes Study (UKPDS) Group. Lancet. 1998; 352(9131): 837-53.

26. Gerstein HC, Bosch J, Dagenais GR, Diaz R, Jung H, Maggioni AP, et al. Basal insulin and cardiovascular and other outcomes in dysglycemia. The New England journal of medicine. 2012; 367(4): 319-28.

27. Budnitz DS, Lovegrove MC, Shehab N, Richards CL. Emergency hospitalizations for adverse drug events in older Americans. The New England journal of medicine. 2011; 365(21): 2002-12.

28. Lipska KJ, Ross JS, Wang Y, Inzucchi SE, Minges K, Karter AJ, et al. National trends in US hospital admissions for hyperglycemia and hypoglycemia among Medicare beneficiaries, 1999 to 2011. JAMA internal medicine. 2014; 174(7): 1116-24.

29. Pearson ER, Donnelly LA, Kimber C, Whitley A, Doney AS, McCarthy MI, et al. Variation in TCF7L2 influences therapeutic response to sulfonylureas: a GoDARTs study. Diabetes. 2007; 56(8): 2178-82.

30. Lyssenko V, Bianchi C, Del Prato S. Personalized Therapy by Phenotype and Genotype. Diabetes care. 2016; 39 Suppl 2: S127-36.

31. Gloyn AL, Weedon MN, Owen KR, Turner MJ, Knight BA, Hitman G, et al. Large-scale association studies of variants in genes encoding the pancreatic beta-cell KATP channel subunits Kir6.2 (KCNJ11) and SUR1 (ABCC8) confirm that the KCNJ11 E23K variant is associated with type 2 diabetes. Diabetes. 2003; 52(2): 568-72.

32. Florez JC, Burtt N, de Bakker PI, Almgren P, Tuomi T, Holmkvist J, et al. Haplotype structure and genotype-phenotype correlations of the sulfonylurea receptor and the islet ATP-sensitive potassium channel gene region. Diabetes. 2004; 53(5): 1360-8.

33. Feng Y, Mao G, Ren X, Xing H, Tang G, Li Q, et al. Ser1369Ala variant in sulfonylurea receptor gene ABCC8 is associated with antidiabetic efficacy of gliclazide in Chinese type 2 diabetic patients. Diabetes care. 2008; 31(10): 1939-44.

34. Hamming KS, Soliman D, Matemisz LC, Niazi O, Lang Y, Gloyn AL, et al. Coexpression of the type 2 diabetes susceptibility gene variants KCNJ11 E23K and ABCC8 S1369A alter the ATP and sulfonylurea sensitivities of the ATP-sensitive K(+) channel. Diabetes. 2009; 58(10): 2419-24.

35. Musso G, Cassader M, Paschetta E, Gambino R. Thiazolidinediones and Advanced Liver Fibrosis in Nonalcoholic Steatohepatitis: A Meta-analysis. JAMA internal medicine. 2017; 177(5): 633-40. 
36. Kernan WN, Viscoli CM, Furie KL, Young LH, Inzucchi SE, Gorman M, et al. Pioglitazone after Ischemic Stroke or Transient Ischemic Attack. The New England journal of medicine. 2016; 374(14): 1321-31.

37. Dawed AY, Donnelly L, Tavendale R, Carr F, Leese G, Palmer CN, et al. CYP2C8 and SLCO1B1 Variants and Therapeutic Response to Thiazolidinediones in Patients With Type 2 Diabetes. Diabetes care. 2016; 39(11): 1902-8.

38. Chiasson JL, Josse RG, Gomis R, Hanefeld M, Karasik A, Laakso M, et al. Acarbose treatment and the risk of cardiovascular disease and hypertension in patients with impaired glucose tolerance: the STOP-NIDDM trial. Jama. 2003; 290(4): 486-94.

39. Maruthur NM, Gribble MO, Bennett WL, Bolen S, Wilson LM, Balakrishnan P, et al. The pharmacogenetics of type 2 diabetes: a systematic review. Diabetes care. 2014; 37(3): 876-86.

40. Holman RR, Coleman RL, Chan JCN, Chiasson JL, Feng H, Ge J, et al. Effects of acarbose on cardiovascular and diabetes outcomes in patients with coronary heart disease and impaired glucose tolerance (ACE): a randomised, double-blind, placebo-controlled trial. The lancet Diabetes \& endocrinology. 2017; 5(11): 877-86.

41. Gerstein HC, Miller ME, Byington RP, Goff DC, Jr., Bigger JT, Buse JB, et al. Effects of intensive glucose lowering in type 2 diabetes. The New England journal of medicine. 2008; 358(24): 2545-59. 42. Shah HS, Gao H, Morieri ML, Skupien J, Marvel S, Pare G, et al. Genetic Predictors of Cardiovascular Mortality During Intensive Glycemic Control in Type 2 Diabetes: Findings From the ACCORD Clinical Trial. Diabetes care. 2016; 39(11): 1915-24.

43. Shah HS, Morieri ML, Marcovina SM, Sigal RJ, Gerstein HC, Wagner MJ, et al. Modulation of GLP1 Levels by a Genetic Variant That Regulates the Cardiovascular Effects of Intensive Glycemic Control in ACCORD. Diabetes care. 2018; 41(2): 348-55.

44. Drucker DJ, Habener JF, Holst JJ. Discovery, characterization, and clinical development of the glucagon-like peptides. The Journal of clinical investigation. 2017; 127(12): 4217-27.

45. Ferrannini E. Sodium-Glucose Co-transporters and Their Inhibition: Clinical Physiology. Cell metabolism. 2017; 26(1): 27-38.

46. Mulvihill EE, Drucker DJ. Pharmacology, Physiology and Mechanisms of Action of Dipeptidyl Peptidase-4 Inhibitors. Endocrine reviews. 2014; 6: 992-1019.

47. Magen D, Sprecher E, Zelikovic I, Skorecki K. A novel missense mutation in SLC5A2 encoding SGLT2 underlies autosomal-recessive renal glucosuria and aminoaciduria. Kidney international. 2005; 67(1): 34-41.

48. Scott RA, Freitag DF, Li L, Chu AY, Surendran P, Young R, et al. A genomic approach to therapeutic target validation identifies a glucose-lowering GLP1R variant protective for coronary heart disease. Science Translational Medicine. 2016; 8(341): 341ra76-ra76.

49. Mahajan A, Sim X, Ng HJ, Manning A, Rivas MA, Highland HM, et al. Identification and functional characterization of G6PC2 coding variants influencing glycemic traits define an effector transcript at the G6PC2-ABCB11 locus. PLoS genetics. 2015; 11(1): e1004876.

50. Wessel J, Chu AY, Willems SM, Wang S, Yaghootkar H, Brody JA, et al. Low-frequency and rare exome chip variants associate with fasting glucose and type 2 diabetes susceptibility. Nature communications. 2015; 6: 5897.

51. t Hart LM, Fritsche A, Nijpels G, van Leeuwen N, Donnelly LA, Dekker JM, et al. The CTRB1/2 locus affects diabetes susceptibility and treatment via the incretin pathway. Diabetes. 2013; 62(9): 327581.

52. Dennis JM, Shields BM, Hill AV, Knight BA, McDonald TJ, Rodgers LR, et al. Precision Medicine in Type 2 Diabetes: Clinical Markers of Insulin Resistance Are Associated With Altered Short- and LongTerm Glycemic Response to DPP-4 Inhibitor Therapy. Diabetes care. 2018. 
53. Aron-Wisnewsky J, Sokolovska N, Liu Y, Comaneshter DS, Vinker S, Pecht T, et al. The advancedDiaRem score improves prediction of diabetes remission 1 year post-Roux-en-Y gastric bypass.

Diabetologia. 2017; 60(10): 1892-902.

54. Adams TD, Davidson LE, Litwin SE, Kim J, Kolotkin RL, Nanjee MN, et al. Weight and Metabolic Outcomes 12 Years after Gastric Bypass. The New England journal of medicine. 2017; 377(12): 1143-55. 55. Sjostrom L, Peltonen M, Jacobson P, Sjostrom CD, Karason K, Wedel H, et al. Bariatric surgery and long-term cardiovascular events. Jama. 2012; 307(1): 56-65.

56. Romeo S, Maglio C, Burza MA, Pirazzi C, Sjoholm K, Jacobson P, et al. Cardiovascular events after bariatric surgery in obese subjects with type 2 diabetes. Diabetes care. 2012; 35(12): 2613-7.

57. Meyer RJ. Precision Medicine, Diabetes, and the U.S. Food and Drug Administration. Diabetes care. 2016; 39(11): 1874-8.

58. Cahn A, Cefalu WT. Clinical Considerations for Use of Initial Combination Therapy in Type 2 Diabetes. Diabetes care. 2016; 39 Suppl 2: S137-45.

59. Zinman B, Wanner C, Lachin JM, Fitchett D, Bluhmki E, Hantel S, et al. Empagliflozin, Cardiovascular Outcomes, and Mortality in Type 2 Diabetes. The New England journal of medicine. 2015; 373(22): 2117-28.

60. Marso SP, Daniels GH, Brown-Frandsen K, Kristensen P, Mann JFE, Nauck MA, et al. Liraglutide and Cardiovascular Outcomes in Type 2 Diabetes. New England Journal of Medicine. 2016; 375: 311-22. 61. Neal B, Perkovic V, Mahaffey KW, de Zeeuw D, Fulcher G, Erondu N, et al. Canagliflozin and Cardiovascular and Renal Events in Type 2 Diabetes. The New England journal of medicine. 2017; 377(7): 644-57.

62. Skyler JS, Bakris GL, Bonifacio E, Darsow T, Eckel RH, Groop L, et al. Differentiation of Diabetes by Pathophysiology, Natural History, and Prognosis. Diabetes. 2017; 66(2): 241-55.

63. Khera AV, Emdin CA, Drake I, Natarajan P, Bick AG, Cook NR, et al. Genetic Risk, Adherence to a Healthy Lifestyle, and Coronary Disease. The New England journal of medicine. 2016; 375(24): 2349-58. 64. Zinman B, Marso SP, Poulter NR, Emerson SS, Pieber TR, Pratley RE, et al. Day-to-day fasting glycaemic variability in DEVOTE: associations with severe hypoglycaemia and cardiovascular outcomes (DEVOTE 2). Diabetologia. 2017.

65. Pieber TR, Marso SP, McGuire DK, Zinman B, Poulter NR, Emerson SS, et al. DEVOTE 3: temporal relationships between severe hypoglycaemia, cardiovascular outcomes and mortality. Diabetologia.

2017.

66. Marx N, Rosenstock J, Kahn SE, Zinman B, Kastelein JJ, Lachin JM, et al. Design and baseline characteristics of the CARdiovascular Outcome Trial of LINAgliptin Versus Glimepiride in Type 2 Diabetes (CAROLINA(R)). Diabetes \& vascular disease research. 2015; 12(3): 164-74.

67. Cusi K, Orsak B, Bril F, Lomonaco R, Hecht J, Ortiz-Lopez C, et al. Long-Term Pioglitazone Treatment for Patients With Nonalcoholic Steatohepatitis and Prediabetes or Type 2 Diabetes Mellitus: A Randomized Trial. Annals of internal medicine. 2016; 165(5): 305-15.

68. Armstrong MJ, Gaunt P, Aithal GP, Barton D, Hull D, Parker R, et al. Liraglutide safety and efficacy in patients with non-alcoholic steatohepatitis (LEAN): a multicentre, double-blind, randomised, placebo-controlled phase 2 study. Lancet. 2016; 387(10019): 679-90.

69. Edelman SV, Polonsky WH. Type 2 Diabetes in the Real World: The Elusive Nature of Glycemic Control. Diabetes care. 2017; 40(11): 1425-32.

70. Carls GS, Tuttle E, Tan RD, Huynh J, Yee J, Edelman SV, et al. Understanding the Gap Between Efficacy in Randomized Controlled Trials and Effectiveness in Real-World Use of GLP-1 RA and DPP-4 Therapies in Patients With Type 2 Diabetes. Diabetes care. 2017; 40(11): 1469-78.

71. Khunti K, Seidu S, Kunutsor S, Davies M. Association Between Adherence to Pharmacotherapy and Outcomes in Type 2 Diabetes: A Meta-analysis. Diabetes care. 2017; 40(11): 1588-96. 
72. Rodgers LR, Weedon MN, Henley WE, Hattersley AT, Shields BM. Cohort profile for the MASTERMIND study: using the Clinical Practice Research Datalink (CPRD) to investigate stratification of response to treatment in patients with type 2 diabetes. BMJ open. 2017; 7(10): e017989.

73. Walford GA, Colomo N, Todd JN, Billings LK, Fernandez M, Chamarthi B, et al. The study to understand the genetics of the acute response to metformin and glipizide in humans (SUGAR-MGH): design of a pharmacogenetic resource for type 2 diabetes. PloS one. 2015; 10(3): e0121553.

74. Srinivasan S, Kaur V, Chamarthi B, Littleton KR, Chen L, Manning AK, et al. TCF7L2 Genetic Variation Augments Incretin Resistance and Influences Response to a Sulfonylurea and Metformin: The Study to Understand the Genetics of the Acute Response to Metformin and Glipizide in Humans (SUGAR-MGH). Diabetes care. 2018. 